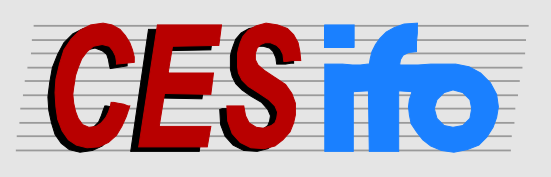

\title{
Working
}

Papers

www.cesifo.org/wp

\section{Hypergeorgism: When is Rent Taxation as a Remedy for Insufficient Capital Accumulation Socially Optimal?}

\author{
Ottmar Edenhofer \\ Linus Mattauch \\ Jan Siegmeier
}

\section{CESIFO WORKING PAPER NO. 4144 \\ CATEGORY 1: Public FinANCE MARCH 2013}

\footnotetext{
An electronic version of the paper may be downloaded

- from the SSRN website:

- from the RePEc website:

- from the CESifo website:

www.SSRN.com

www.RePEc.org

www.CESifo-group.org/wp
}

\section{CESifo}




\title{
Hypergeorgism: When is Rent Taxation as a Remedy for Insufficient Capital Accumulation Socially Optimal?
}

\begin{abstract}
Imperfect altruism between generations may lead to insufficient capital accumulation. We study the welfare consequences of taxing the rent on a fixed production factor, such as land, in this setting. We prove that taxing the rent is welfare-enhancing as it increases capital investment. This holds for any tax level and any recycling of the tax revenues except for combinations of high taxes and strongly redistributive recycling. Specific forms of redistribution of the land rent tax - a capital subsidy or a transfer directed at fundless newborns - allow to reproduce the social optimum under parameter restrictions valid for most economies.
\end{abstract}

JEL-Code: H210, H220, H230, Q240.

Keywords: land rent tax, overlapping generations, revenue recycling, social optimum, underaccumulation.

\author{
Ottmar Edenhofer \\ Potsdam Institute for Climate Impact \\ Research \\ Postbox 601203 \\ Germany - 14412 Potsdam \\ ottmar.edenhofer@pik-potsdam.de
}

\author{
Linus Mattauch \\ Mercator Research Institute on Global \\ Commons and Climate Change \\ Berlin / Germany \\ mattauch@mcc-berlin.de
}

\author{
Jan Siegmeier \\ Technical University of Berlin and \\ Mercator Research Institute on Global Commons \\ and Climate Change \\ Torgauer Strasse 12 - 15 \\ Germany - 10829 Berlin \\ jan.siegmeier@mailbox.tu-berlin.de
}

February 18, 2013

Authors are listed alphabetically as all contributed equally to the paper. 


\section{Introduction}

A central thesis of public economics is that taxation for the purpose of redistribution leads to a loss in efficiency: While revenue from taxing the income of the owners of productive assets can for example be used to improve the living conditions of the less wealthy, it is usually considered to reduce economic output.

In this paper we disprove this thesis for the case that a fixed production factor is economically relevant and that imperfect altruism between generations leads to underaccumulation. We show that taxing the rents on a fixed production factor increases total consumption and welfare even if the tax revenues are used to compensate households for their respective tax payments. We then demonstrate that other redistribution patterns, such as a uniform transfer, increase productivity further. Giving a disproportionately high share of the tax revenue to the poor younger generations even allows to reproduce the social optimum by making the productivity loss vanish, but can lead to overaccumulation if the land rent tax is very high. Similar outcomes can be achieved by a capital subsidy.

Our argument is based on two premises. First, we make minimal assumptions about household heterogeneity: There are no bequests, so individuals are differentiated by age because they are born fundless and accumulate wealth over time. No further structure such as a lifecycle of working and retirement is imposed. This assumption implies that capital accumulation is suboptimally low compared to the case of perfect altruism between all generations.

Second, fixed factors of production matter for the size of economic output (see Caselli and Feyrer 2007 for an analysis of the role of land and natural resources). Land scarcity is ubiquitous in explaining economic outcomes, from the real estate to the agricultural sector. Although land is the canonical example for a fixed production factor, it is not the only one. The stocks of old industries in which no further investment will be undertaken are best understood as fixed production factors and exogenously given availability of natural resources is another example.

The central tenet of georgism George 1879, Heavey 2003 is that taxing rents from such fixed production factors is a way of raising fiscal income that is non-distortionary. Feldstein argued that this is not the case if owners of land also provide other factors such as capital, if there is an alternative in saving decisions to invest in land or capital or if these assets have different risks [Feldstein 1977]. While we confirm that taxing fixed factors is not neutral in a model with alternative assets and can increase aggregate capital and consumption [Petrucci 2006], our contribution is that a tax on such rents is actually welfare-enhancing and can even be socially optimal. The underlying mechanism is composed of two effects: On the one hand, the tax shifts investment towards reproducible stocks, alleviating their undersupply 
and leading to higher output and aggregate consumption. On the other hand, redistributing the tax revenue to those generations in the economy whose utility will benefit most from additional funds creates an additional positive welfare effect. Extending and modifying the tenet of georgism, we propose that this insight be called hypergeorgism 1

Our finding has implications for interpreting the relationship between land prices and economic growth: the fixed factor land attracts more wealth than optimal, causing underinvestment in reproducible stocks such as capital. Thus high land prices might be an indicator of too little investment in capital. If capital accumulation is suboptimal, land taxation can hence be a remedy as more accumulation of reproducible factors is induced. Such a mechanism may explain that during the recent financial and currency crises, partly caused by a housing bubble, higher investments in real estate and agricultural goods exacerbated the challenge of economic stabilization. A remedy could be to stimulate the economy by taxing land rents.

In Section 2, a continuous overlapping generations (OLG) model is introduced to study the relationship between househould heterogeneity and the trade-off of investing in land or reproducible assets: we thus derive a well-known arbitrage condition for investments into capital or land. The aggregate consumption growth - compared to the standard OLG outcome - is additionally influenced by the land price and the type of redistribution of the tax revenue. As the benchmark for tax policy evaluation, we take social welfare to be the preference satisfaction of all heterogeneous individuals Calvo and Obstfeld 1988.

In Section 3, the main result is proved: Any redistribution of the revenue from land rent taxation that is stable in the long run and not too egalitarian stimulates capital accumulation and thus increases total consumption and welfare (Theorem 4). If a disproportionate share of the revenue is given to the young generations, the social optimum can be achieved, but overaccumulation is possible if the tax rate is high (Theorem 5). Furthermore, the fiscally realistic case of a uniform age- and wealth-independent redistribution is shown to be more efficient than the case of a wealth-neutral redistribution; but both are never socially optimal (Sections 3.3.2 and 3.3.5). We also calculate conditions under which two specific policies are socially optimal: the redistribution of the land tax revenue to the newborn generation only (or alternatively redistributing it exponentially with age, see Sections 3.3 .3 and 3.3.4 and the use of the revenue as a capital subsidy (Section 3.4). The results about the different possible redistributions are summarized in Table 1.

In Section 4 the application of our formal result to fiscal policy is dis-

\footnotetext{
${ }^{1}$ From a historical perspective, our result may be closer to Henry George's original thinking than georgism or the neoclassical Henry George Theorems: Henry George was chiefly concerned with poverty eradication George 1879. We show that taxing rent income and giving it to the poor young generations actually enhances the economic efficiency.
} 
cussed: we find that the conditions for social optimality plausibly hold for the economies of a large and diverse set described in an empirical survey. Furthermore we justify our choice of social optimum.

The present study builds on two strands of literature. The relationship between the land price, the land rent and the interest rate has been discussed by Feldstein 1977, Calvo, Kotlikoff and Rodriguez 1979, Burgstaller 1994, Foley and Michl 1999]. Chamley and Wright 1987 studied the dynamic effects of fiscal policy in this context. On the other hand, in the continuous overlapping generations model of Blanchard Blanchard 1985, land has been introduced as a production factor [Buiter 1989] to study when in such a model Ricardian equivalence holds [Buiter 1988, Weil 1989]. Buiter was to the best of our knowledge the first to consider the impact of land as a fixed production factor in the continuous OLG model. He proved that with land Ricardian equivalence holds despite the arrival of newborn generations [Buiter 1989], but did not take into account capital accumulation. Fried and Howitt 1988] also considered the interaction of debt with land for the case of an open economy. The question of determining the socially optimal allocation in a continuous OLG model has been treated by Calvo and Obstfeld 1988.

The work that is closest to our study is [Petrucci 2006], which uses a model similar to that presented in this article to discuss the impact of an endogenous labour-leisure choice on the incidence of a land rent tax. Petrucci states that a land rent tax leads to higher capital and consumption, but does not consider the welfare effects and the redistribution possibilities of the land tax revenue. Hashimoto and Sakuragawa 1998 study the welfare consequences of a land rent tax in a discrete OLG model with endogenous technological change. They also find that it is preferable to redirect the revenue to the young generation, but in their model neither a Pareto improvement nor a socially optimal allocation can be reached.

\section{Model}

We extend the continuous overlapping generations (OLG) model of Yaari and Blanchard [Yaari 1965, Blanchard 1985, to include a fixed factor of production, which we label land Buiter 1989, Foley and Michl 1999 22 Our model describes an economy with one final good of unit price and three other flow markets for labour, capital and land rental as production inputs as well as two stock markets for capital and land ownership. We first describe the economy's demography, the individuals' maximization problem and budget constraint and the role of the government. The following four subsections detail individual households' behavior (2.1), aggregate quanti-

\footnotetext{
${ }^{2}$ Whenever we mention a "standard" result below, this refers to the model of Blanchard 1985.
} 
ties 2.2 , the simple production structure of the model 2.3 ) and the social planner solution 2.4. In these sections the important conditions are summarized, full derivations are found in the appendix.

Assume a constant birth rate $\phi$, equal to each individual's probability of death in the next period and thus to the death rate in a large population. Therefore population size is constant - henceforth normalized to 1 - and individuals' lifetimes are exponentially distributed. The size at time $\tau$ of a cohort born at time $\nu$ is $\phi e^{-\phi(\tau-\nu)}$.

An individual born at time $\nu$ with a rate of pure time preference $\rho$ and instantaneous utility $\ln c(\nu, t)$ has an expected lifetime utility $u(\nu, t)$ at time $t \geq \nu$ given by

$$
u(\nu, t)=\int_{t}^{\infty} \ln c(\nu, \tau) e^{-(\phi+\rho)(\tau-t)} \mathrm{d} \tau
$$

with $c(\nu, \tau)$ describing the path of consumption. In the following, "utility" refers to $u(\nu, t)$ unless otherwise noted.

At time $\tau$, each individual supplies one unit of labour and receives an age-independent wage $w$. Individuals born at $\nu$ own capital $k(\nu, \tau)$ and a share $s(\nu, \tau)$ of total land $\bar{S}$, which can be bought and sold at a price $p(\tau)$. Total land is constant and all land is owned by somebody:

$$
\int_{-\infty}^{\tau} s(\nu, \tau) \phi e^{-\phi(\tau-\nu)} \mathrm{d} \nu=S(\tau)=\bar{S} \quad \text { const. }
$$

Individuals rent out their capital and land to firms at market rates $r(\tau)$ and $l(\tau)$, respectively, and have no bequest motives. Instead, to maximize utility from their savings under lifetime uncertainty, they use life-insurance contracts (as in the standard case). The insurance companies top up interest income by an annuity in return for obtaining the individual's financial assets in case of death. If the insurance sector is competitive and has no cost, all financial wealth of those who died is redistributed to the living in proportion to their capital and the annuity is $\phi k(\nu, \tau)$. Similarly, insurance companies distribute land $\phi s(\nu, \tau)$ to individuals born at $\nu$ in proportion to their land ownership, in return for receiving their land in case of death. Thus, the changes in land ownership of all living generations do not sum to zero:

$$
\int_{-\infty}^{\tau} \dot{s}(\nu, \tau) \phi e^{-\phi(\tau-\nu)} \mathrm{d} \nu=\phi \bar{S} .
$$

The government levies a tax $T(\tau)$ on land rents and instantaneously redistributes the full revenue to individuals via potentially age-dependent transfers $\gamma(\nu, \tau)$. 
Definition 1. A redistribution scheme $\gamma(\nu, \tau)$ is called permissible if it is non-negative for all $\nu$ and $\tau$ and satisfies the government budget identity at all times:

$$
\int_{-\infty}^{\tau} \gamma(\nu, \tau) \phi e^{-\phi(\tau-\nu)} \mathrm{d} \nu=T(\tau) l(\tau) \bar{S} \quad \text { for all } \tau
$$

The following analysis is restricted to permissible redistribution schemes: the government budget is balanced at all times, so there is no government debt.

Individuals thus have the following budget identity, valid for all $\tau \in[t, \infty):$

$$
\begin{aligned}
\dot{k}(\nu, \tau)+p(\tau) \dot{s}(\nu, \tau) & =w(\tau)+[r(\tau)+\phi] k(\nu, \tau)+ \\
& +[[1-T(\tau)] l(\tau)+p(\tau) \phi] s(\nu, \tau)+\gamma(\nu, \tau)-c(\nu, \tau)
\end{aligned}
$$

where $\dot{k}(\nu, \tau)=\mathrm{d} k(\nu, \tau) / \mathrm{d} \tau$, etc.

The individual also respects a solvency condition which prevents her from playing a Ponzi-game against the life-insurance companies:

$$
\begin{array}{ll} 
& \lim _{\tau \rightarrow \infty}[k(\nu, \tau)+p(\tau) s(\nu, \tau)] e^{-R(t, \tau)}=0 \\
\text { with } & R(t, \tau) \equiv \int_{t}^{\tau}(r(\tilde{t})+\phi) \mathrm{d} \tilde{t} .
\end{array}
$$

Although the individual can take up debt $(k(\nu, \tau)<0)$, the limit of the present value of her total financial and land wealth at infinity has to be zero. Note that there can be no debt in terms of land, so land appears as a collateral for capital debt in the transversality condition and its present value is evaluated using the capital interest rate.

\subsection{Individual households' solution and arbitrage condition}

Individuals maximize utility defined in Equation (1) by choosing $c(\nu, \tau)$ and $s(\nu, \tau)$, subject to Equation (5) and the transversality condition (6). From the first-order conditions of this optimization problem, one obtains the usual Keynes-Ramsey rule for the dynamics of individual consumption

$$
\frac{\dot{c}(\nu, \tau)}{c(\nu, \tau)}=r(\tau)-\rho
$$

and the arbitrage condition Burgstaller 1994, Foley and Michl 1999 between land and capital:

$$
\frac{[1-T(\tau)] l(\tau)}{p(\tau)}+\frac{\dot{p}(\tau)}{p(\tau)}=r(\tau) .
$$


For the derivation of the two conditions, see Appendix (6.1). The arbitrage condition is crucial for the main result below since it links the stock and flow markets for land by relating the unit value of land as an investment $p$ to its after-tax rent, $(1-T) l$.

Using the instantaneous budget identity (5), the transversality condition (6) and the arbitrage condition (8), a lifetime budget constraint can be derived (see Appendix 6.2). It states that the present value of the consumption plan at time $t$ of individuals born at $\nu$ equals their total wealth consisting of capital, land and the present values of lifetime labor income and transfers:

$$
\begin{aligned}
\int_{t}^{\infty} c(\nu, \tau) e^{-R(t, \tau)} \mathrm{d} \tau & =k(\nu, t)+p(t) s(\nu, t)+H(t)+\bar{\gamma}(\nu, t) \\
\text { where } H(t) & \equiv \int_{t}^{\infty} w(\tau) e^{-R(t, \tau)} \mathrm{d} \tau \\
\text { and } \bar{\gamma}(\nu, t) & \equiv \int_{t}^{\infty} \gamma(\nu, \tau) e^{-R(t, \tau)} \mathrm{d} \tau .
\end{aligned}
$$

Solving the Keynes-Ramsey rule (7) for $c(\nu, \tau)$ and using the result in Equation (9) shows that all individuals consume the same fixed fraction of their total wealth consisting of capital, land and the present value of lifetime labor income and transfers:

$$
c(\nu, t)=(\rho+\phi)[k(\nu, t)+p(t) s(\nu, t)+H(t)+\bar{\gamma}(\nu, t)]
$$

(for the derivation see Appendix 6.2). Equations (9) and (10) differ from the standard results only by the tax redistribution terms and the $p(\tau) s(\nu, \tau)$ terms due to the inclusion of land in our model.

\subsection{Aggregation}

Aggregation of an individual variable $x$ to its equivalent for the total population $X$ is defined by

$$
X(t)=\int_{-\infty}^{t} x(\nu, t) \phi e^{-\phi(t-\nu)} \mathrm{d} \nu
$$

Employing Equation (2) for total land, we obtain from aggregation of Equation 10$)$ :

$$
\begin{aligned}
C(t) & =(\rho+\phi)[K(t)+p(t) \bar{S}+H(t)+\bar{\Gamma}(t)], \\
\text { with } \quad \bar{\Gamma}(t) & \equiv \int_{-\infty}^{t} \bar{\gamma}(\nu, t) \phi e^{-\phi(t-\nu)} \mathrm{d} \nu
\end{aligned}
$$

and with $C(t)$ and $K(t)$ denoting total consumption and capital. The expression $\bar{\Gamma}(t)$ is the total present value of transfers from the government to 
individuals. Therefore aggregate consumption is the same constant fraction of total wealth as for each individual.

Using Leibniz' rule on the general aggregation formula applied to capital and the individual budget constraint (5), the dynamics of the total capital stock can be derived (see Appendix (6.3)):

$$
\dot{K}(t)=w(t)+r(t) K(t)+l(t) \bar{S}-C(t) .
$$

This resembles the standard result apart from the land-related term. Note that taxes and transfers do not appear in this expression, as they cancel out for the dynamics of aggregate capital.

Finally, again applying Leibniz' rule on the aggregation expression for consumption and using Equations (7) and (10), one obtains the dynamics of aggregate consumption (see Appendix (6.3)):

$$
\frac{\dot{C}(t)}{C(t)}=r(t)-\rho-\phi(\rho+\phi) \frac{K(t)+p(t) \bar{S}+\bar{\Gamma}(t)-\bar{\gamma}(t, t)}{C(t)} .
$$

The last term reflects the "generation replacement effect": A fraction $\phi$ of the total population, owning aggregate capital $K(t)$ (as in the standard model) and land wealth $p \bar{S}$ and expecting lifetime transfers of $\bar{\Gamma}(t)$, dies and is replaced by newborns, whose only non-human wealth consists of expected lifetime transfers $\bar{\gamma}(t, t)$. Since individuals consume a fixed fraction $(\rho+\phi)$ of their wealth, this continuous turnover affects aggregate consumption growth. Growth is diminished by the newborns' missing capital and land but also impacted (positively or negatively) by future transfer payments, depending on how these redistribute wealth between generations. We will come back to this mechanism in Section 3.2 .

\section{$2.3 \quad$ Firms}

Assume a single final good is produced from inputs $K, L$ and $S$ with a production function featuring constant returns to scale, diminishing marginal productivity in individual inputs and satisfying the Inada conditions in all three arguments. The representative firm's problem is

$$
\max _{K(t), L(t), S(t)} F(K(t), L(t), S(t))-R(t) K(t)-w(t) L(t)-l(t) S(t)
$$

yielding the standard first-order conditions

$$
\begin{aligned}
R(t) & =F_{K}(K(t), L(t), S(t)), \\
w(t) & =F_{L}(K(t), L(t), S(t)), \\
l(t) & =F_{S}(K(t), L(t), S(t)) .
\end{aligned}
$$

With a depreciation rate of private capital $\delta$, the market interest rate is then given by

$$
r(t)=R(t)-\delta
$$




\subsection{Social planner}

The social planner solution is chosen as a normative benchmark to evaluate the tax policies suggested below. We assume that social welfare is the preference satisfaction of all heterogeneous individuals where the socially optimal discount rate is the private discount rate. Once the optimal static distribution is chosen, the intertemporally optimal solution can be chosen separately. This insight is provided by the Calvo-Obstfeld two-step procedure for evaluating social welfare in economies with overlapping generations Calvo and Obstfeld 1988]. For this approach to justify the social optimum, define $U(C(t))$ as the optimal solution to the static maximization problem:

$$
\begin{aligned}
U(C(t)) & =\max _{\{c(\nu, t)\}_{\nu=-\infty}^{t}} \int_{-\infty}^{t} \ln c(\nu, t) \phi e^{-\phi(t-\nu)} \mathrm{d} \nu \\
\text { subject to: } \quad C(t) & =\int_{-\infty}^{t} c(\nu, t) \phi e^{-\phi(t-\nu)} \mathrm{d} \nu .
\end{aligned}
$$

Solving this optimal control problem with an integral constraint, it can be found that

$$
U(C(t))=\ln (C(t)) .
$$

The intertemporal maximization problem of the social planner is hence the following Ramsey-type problem:

$$
\begin{aligned}
\max _{C(t)} & \int_{t=0}^{\infty} U(C(t)) e^{-\rho t} d t \\
\text { with } \quad U(C) & =\ln (C) \\
\text { s.t. } \quad \dot{K}(t) & =F(K(t), L(t), S(t))-C(t)-\delta K(t) .
\end{aligned}
$$

The corresponding rule for socially optimal aggregate consumption growth is thus the Keynes-Ramsey rule

$$
\frac{\dot{C}(t)}{C(t)}=F_{K}(K(t), L(t), S(t))-\delta-\rho .
$$

Being in this paper only concerned with policy measures that raise total consumption, we ignore the question of its static distribution: this amounts to defending the position that when social welfare is increased, it is permissible that some individuals lose if others gain more. Thus a policy measure counts as welfare-improving if it yields a Kaldor-Hicks, but not a Pareto improvement for the generations alive at a particular date. (Implications of adopting this normative viewpoint are discussed in see Section 4.2.)

As an alternative conception of social welfare, the preference-satisfaction approach is equivalent to assuming the social structure of perfect altruism 
between generations: a single dynasty of households with no fundless newborns. From the perspective of a social planner maximizing social welfare, there is no distinction between individuals, so if the death rate equals the birth rate the replacement dynamics can be ignored.

\section{Results}

We characterise the steady state of our model: Without policy measures, there is underaccumulation of capital (Section 3.1). This leads to our central proposition that taxing land rents is welfare-increasing, for any redistribution scheme yielding a steady state (Section 3.2). We derive a general condition for socially optimal redistributions and then consider specific cases: land rents are redistributed by transfers that are either uniform, positive only for newborns, exponential functions of age or wealth-neutral (Section 3.3. Finally, we consider a capital subsidy as an alternative to redistribution (Section 3.4). Table 1 summarizes the welfare properties of the different possibilities for spending the land tax revenue.

\subsection{Properties of the steady state}

Since $L$ and $S$ are fixed, we drop them as arguments from the production function in the following. The social planner's system is in a steady state if the capital stock and consumption level satisfy

$$
\begin{aligned}
\dot{K} & =0 \rightarrow \quad C^{k r}=F\left(K^{k r}\right)-\delta K^{k r} \\
\dot{C} & =0 \rightarrow 0=F_{K}\left(K^{k r}\right)-\delta-\rho
\end{aligned}
$$

as is well-known. These equations characterize the optimal Keynes-Ramsey levels, denoted by superscripts ${ }^{k r}$, to which we compare the decentralized outcome: Coupled differential equations for the aggregate capital stock (12), aggregate consumption (13) and the land price (8) govern the decentralized system's dynamics ${ }^{3}$ The steady state conditions are obtained by setting the time derivatives to zero and inserting the conditions on prices (15)-(17):

$$
\begin{aligned}
\dot{K} & =0 \rightarrow \quad C_{P}(K)=F(K)-\delta K \\
\dot{C}=0 \rightarrow \quad C_{H}(K) & =\phi(\rho+\phi) \frac{K+p(K) \bar{S}+\bar{\Gamma}(K)-\bar{\gamma}^{N}(K)}{r(K)-\rho} \\
\dot{p}=0 \rightarrow \quad p(K) & =\frac{(1-T) l(K)}{r(K)} .
\end{aligned}
$$

The subscripts ${ }_{P}$ and ${ }_{H}$ highlight that the first equation defines a curve in the $C$ - $K$-plane shaped like a parabola and the second a hyperbola (compare

\footnotetext{
${ }^{3}$ Any specific redistribution $\gamma(\nu, t)$ is expressed in terms of $K, C$ and $p$ and their time derivatives, so $\Gamma$ and $\gamma$ are not independent dynamic variables themselves.
} 
Heijdra 2009, p.572f]; exceptions are discussed below). The present value of current and future transfers to the newborn is denoted by $\bar{\gamma}^{N}(K)=\bar{\gamma}(t, t)$. A unique (non-trivial) steady state solution exists and the steady state is saddle-point stable. In the following the system is reduced to two dimensions by setting $\dot{p}=0$. This projection captures all relevant dynamics. 4 We denote variables at the steady state (where all three of Equations (22) - 24) hold) by an asterisk *, in particular,

$$
\begin{aligned}
p^{*} & =p\left(K^{*}\right)=\frac{(1-T) l\left(K^{*}\right)}{r\left(K^{*}\right)} \\
r^{*} & =r(K)=F_{K}(K)-\delta \\
l^{*} & =l(K)=F_{S}(K) .
\end{aligned}
$$

Later, the position of the hyperbola $(23)$ will be discussed for values of $K$ outside the steady state as if these capital levels were steady states, thus assuming that $r(K)$ is constant (along with $\dot{p}=0$ ). We then write $\bar{\Gamma}^{\dagger}(K)$ and $\bar{\gamma}^{N \dagger}(K)$.

Equation $(23)$ is essential for analyzing the welfare effects of policies. Consider two cases: First, without taxes and transfers $(T=0$ and $\gamma=0)$ it follows that

$$
r\left(K^{*}\right)=\rho+\phi(\rho+\phi) \frac{K^{*}+p\left(K^{*}\right) \bar{S}}{C^{*}} .
$$

Comparing this to the corresponding equation for the social planner (21), it can be seen that the interest rate of the decentralized case is higher than the implied price of capital in the social planner's steady state. From $F_{K K}<0$ and Equations (15) and (18) it follows that $K^{*}<K^{k r}$. As $K^{k r}$ is to the left of the maximum of the parabola described by Equation (22), and thus $C\left(K^{*}\right)<C\left(K^{k r}\right)$, there is suboptimal underaccumulation in the decentralized equilibrium.

Second, with positive taxes and transfers, there are two competing effects entering Equation (23): The wealth loss of land owners due to land rent taxation $\operatorname{Tl}\left(K^{*}\right) \bar{S} / r\left(K^{*}\right)$ and the overall redistribution effect $\bar{\Gamma}^{*}-\bar{\gamma}^{N *}$.

For the aggregated present values of transfers $\bar{\Gamma}^{*}$, a general condition can be derived:

Lemma 2. In a steady state, permissible redistribution schemes satisfy

$$
\phi \bar{\gamma}^{N *}+r\left(K^{*}\right) \bar{\Gamma}^{*}=T l\left(K^{*}\right) \bar{S} .
$$

\footnotetext{
${ }^{4}$ This can be shown in the three-dimensional system: Linearizing around the steady state shows that it is a saddle point with one stable arm. Since $C$ is a jump variable which instantaneously adjusts such that the optimality and transversality conditions are observed, the system is on the stable path.
} 
Proof. We require $d \bar{\Gamma}^{*}(t) / d t=0$ in the steady state. Applying Leibniz' rule leads to the result, see Appendix 6.4 for details 5

An example for a redistribution that is permissible according to Equation (4), but not consistent with a steady state, is a scheme where only generations born before a certain fixed date receive transfers.

The following corollary prepares the main result:

Corollary 3. The aggregate of individual present values of future transfers in the steady state for a permissible redistribution scheme satisfies

$$
\bar{\Gamma}^{*}<\frac{T l\left(K^{*}\right) \bar{S}}{r\left(K^{*}\right)} .
$$

Proof. For a permissible redistribution scheme, we have $\bar{\gamma}^{N *}>0$ in the steady state. The result then follows directly from Equation (27).

Finally, note that in the steady state the growth factor $R(t, \tau)$ simplifies to

$$
R(t, \tau)=\int_{t}^{\tau}(r(\tilde{t})+\phi) \mathrm{d} \tilde{t}=\left(r\left(K^{*}\right)+\phi\right)(\tau-t) .
$$

This simplification will be used for the rest of the article whereever steadystate properties are discussed.

\subsection{Hypergeorgism Theorem}

The main result of the present article is that land rent taxation is efficiencyenhancing as long as the redistribution does not stimulate the capital accumulation too much. This result is split up into two statements that detail the role of the redistribution pattern chosen.

Theorem 4 (Hypergeorgism, basic version). A fiscal policy consisting of taxing land rents and a permissible redistribution scheme yielding a steady state that fulfills $\bar{\Gamma}^{\dagger}(K)-\bar{\gamma}^{N \dagger}(K) \geq 0$ for all $K \in\left[0, K^{k r}\right]$ increases social welfare.

The basic theorem is implicit in [Feldstein 1977] and explicit, but not formalized, in Petrucci 2006 and its welfare consequences as well as the dependency on the redistribution pattern are also not detailed there. It applies to important specific redistributions: a uniform transfer to every citizen, independent of age, and a wealth-neutral transfer such that rentiers are repaid their rent and thus taxes are fully compensated (see Sections 3.3 .2 and 3.3.5). The following more general version is necessary to understand with which redistributions the social optimum can be reached and when too

\footnotetext{
${ }^{5}$ We are indebted to Dankrad Feist for suggesting this calculation.
} 
much redistribution would lead to overaccumulation (see Sections 3.3.3 and 3.3.4):

Theorem 5 (Hypergeorgism, general version). A fiscal policy consisting of taxing land rents and a permissible redistribution scheme yielding a steady state with $\bar{\Gamma}^{\dagger}(K)-\bar{\gamma}^{N^{\dagger}}(K)<0$ for some $K$ increases social welfare if $T \leq \frac{\phi}{\phi+\rho} \sqrt{6}$. For higher $T$, suboptimal overaccumulation of capital is possible, depending on the particular redistribution.

The intuition for the results is as follows: the tax on land rents makes investment in land less attractive, relative to investment in capital. This price effect reduces the underaccumulation of capital, leading to higher output. On the other hand, capital accumulation is also affected by recycling the tax revenues as transfers to individuals. The size and sign of the transfers' effect on aggregate consumption and investment depends on the specific redistribution scheme. As long as the newborns do not receive higher transfers than the average, the redistribution effect is inessential and welfare is increased by the price effect (Figure 1). If the distribution is tilted further towards the young, the increased redistribution effect may shift the aggregate steady state capital stock and consumption to even higher values (Figure 2). If the tax is high at the same time, both effects together may result in overaccumulation (Figure 3).

Proof of Theorem 4. The idea of the proof is to compare the steady state of the system with no policy to that of the policy case: It will be shown that although for a fixed capital stock, consumption is lower with the policy, both consumption and capital stock are higher in the steady state of the policy case. This is illustrated in Figure 1 .

Consider two cases, one without taxes and the other with a land rent taxation level $T>0$. Denote the steady states defined by Equations (22) and (23) for the two cases by $\left(K^{0 *}, C^{0 *}\right)$ and $\left(K^{1 *}, C^{1 *}\right)$ and let the superscripts 0 and 1 also indicate the policy and no-policy case for the parabola and the hyperbola. From the definition of the social planner solution in Section 2.4. it follows that for an increase in social welfare it is sufficient to prove that

$$
C^{0 *}<C^{1 *} \text {. }
$$

The parabola defined by $\dot{K}=0$ is unaffected by taxes and transfers, but the hyperbola, defined by $\dot{C}=0$, changes: Equation 23 can be rewritten as

$$
C_{H}^{1}(K)=\phi \frac{\rho+\phi}{r(K)-\rho}\left\{K+\frac{l(K) \bar{S}}{r(K)}-\frac{T l(K) \bar{S}}{r(K)}+\bar{\Gamma}^{\dagger}(K)-\bar{\gamma}^{N \dagger}(K)\right\},
$$

\footnotetext{
${ }^{6}$ In fact, under some regularity conditions on higher derivatives of the production function, which are for example satisfied by a Cobb-Douglas function, it follows that even $T \leq \phi \frac{\rho K^{k r}+l\left(K^{k r}\right) \bar{S}}{\rho l\left(K^{k r}\right) \bar{S}}$ is sufficient, but this is not true for the general production function considered in this paper.
} 


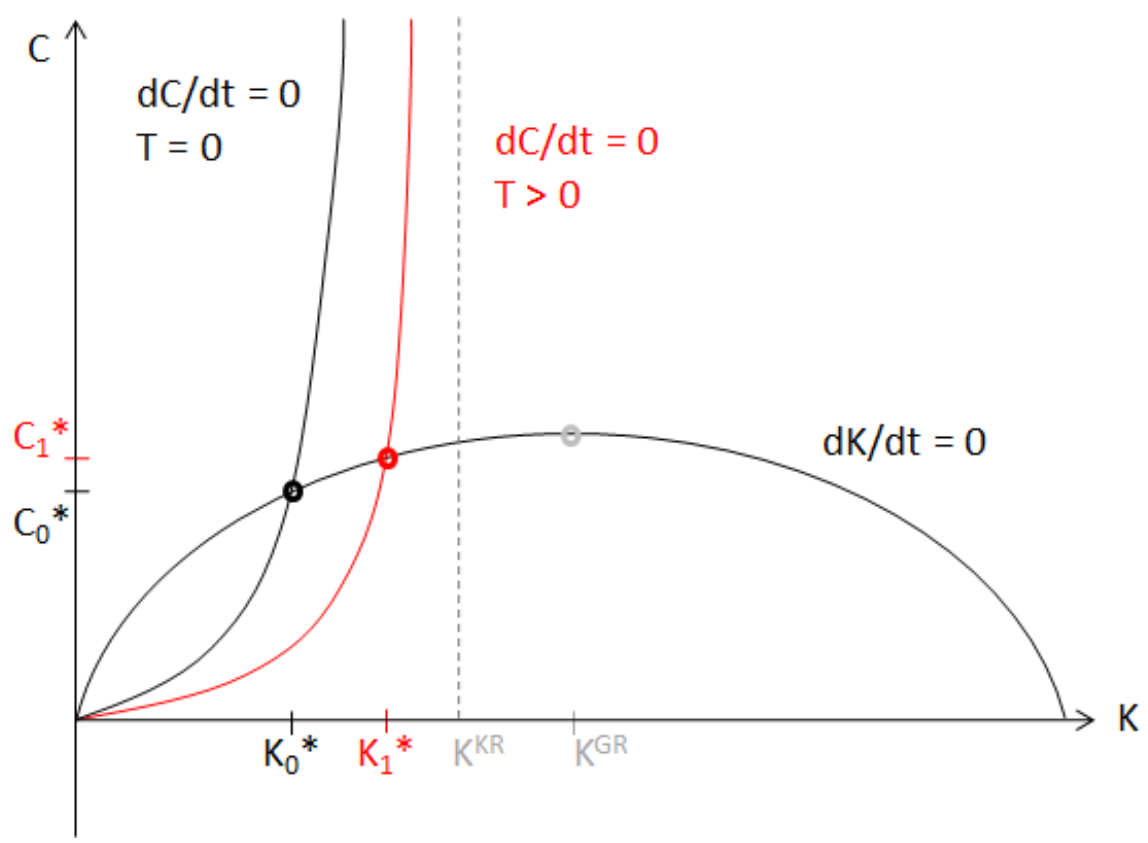

Figure 1: Phase diagram for redistributions with $\bar{\Gamma}^{\dagger}(K)-\bar{\gamma}^{N \dagger}(K) \geq 0$ for all $K \in\left[0, K^{k r}\right]$ and all $T \in[0 ; 1]$

where we treat any value of $K$ as if it was the steady state value (hence the ${ }^{\dagger}$-notation). Corollary 3 can then be written as $\bar{\Gamma}^{\dagger}(K)<\frac{T l(K) \bar{S}}{r(K)}$. Together with the assumption that $\bar{\Gamma}^{\dagger}(K)-\bar{\gamma}^{N \dagger}(K) \geq 0$ for all $K \in\left[0, K^{k r}\right]$, this implies that the last three (the directly policy-dependent) terms in the curly bracket together are negative, and thus that $C_{H}^{1}(K)<C_{H}^{0}(K)$ for all $K \in$ $\left[0, K^{k r}\right]$. In Figure 1, the hyperbola for $T>0$ is below the no-tax case.

For any $K<K^{0 *}$, we also have $C_{H}^{0}(K)<C_{P}^{0}(K)$ and $C_{P}^{0}(K)=C_{P}^{1}(K)$ since the parabola is policy-independent, so $C_{H}^{1}(K)<C_{P}^{1}(K)$ for $K<K^{0 *}$. As $\bar{\Gamma}^{\dagger}(K) \geq \bar{\gamma}^{N \dagger}(K)$ and $T \leq 1, C_{H}^{1}$ is positive for all $K \leq K^{k r}$. Hence the (non-trivial) intersection of parabola and hyperbola for $T_{1}$ must occur at a capital stock $K^{1 *}$ with $K^{0 *} \leq K^{1 *}<K^{k r}$. In this interval, $C_{P}(K)$ is increasing in $K$, thus $K^{0 *}<K^{1 *}$ and also $C^{0 *}<C^{1 *}$, as required.

Proof of Theorem 5. It is sufficient to show that for $T \leq \frac{\phi}{\phi+\rho}$,

$$
K+(1-T) \frac{l(K)}{r(K)} \bar{S}+\bar{\Gamma}^{\dagger}(K)-\bar{\gamma}^{N \dagger}(K) \geq 0
$$

for all $K \geq K^{k r}$ because the intersection of parabola and hyperbola then occurs for $K^{1 *} \leq K^{k r}$ (see Figures 2 and 3). The argument in the previous 


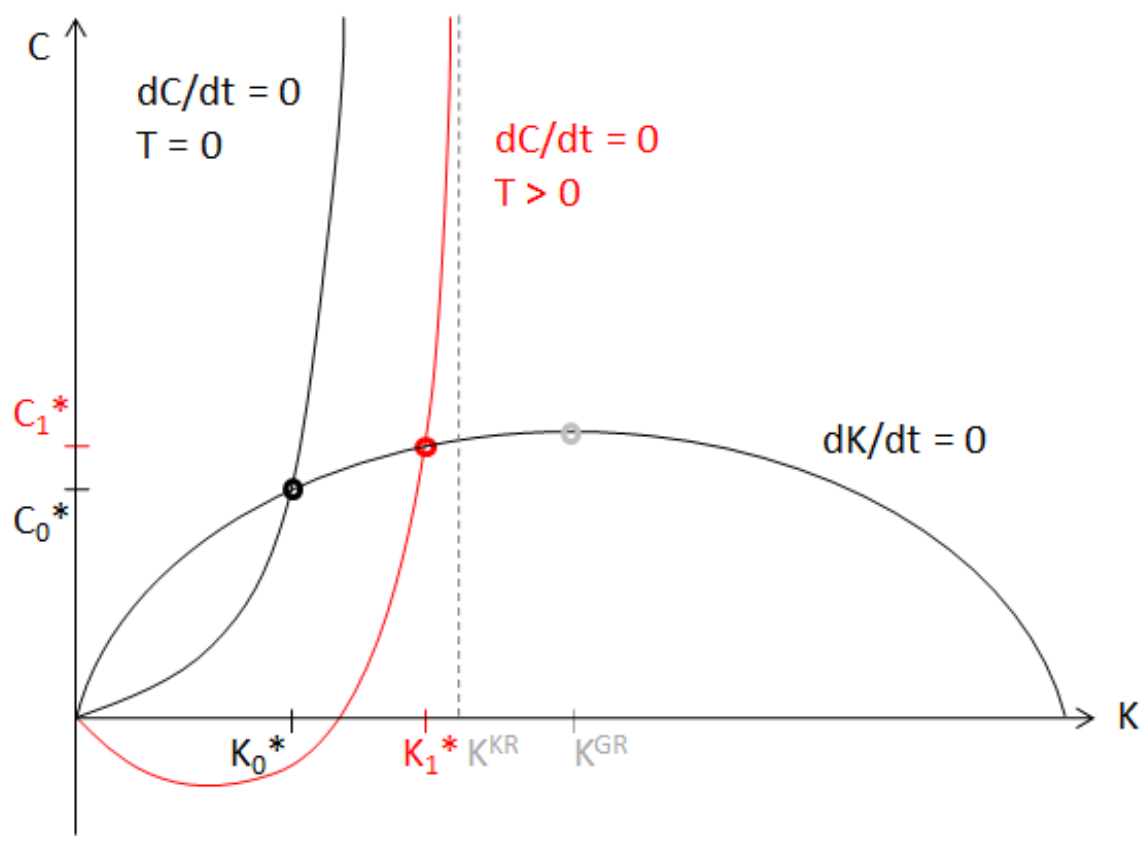

Figure 2: Phase diagram for redistributions with $\bar{\Gamma}^{\dagger}(K)-\bar{\gamma}^{N \dagger}(K)<0$ for some $K \in\left[0, K^{k r}\right]$ and $T<T^{o p t}$

proof is then valid because it is not based on how negative the directly policy dependent terms in Equation (29) are, as long as the numerator is positive. Again, we treat any value of $K$ as if it was the steady state value, highlighted by the ${ }^{\dagger}$-notation. thus

From Lemma 2 it follows that in the steady state $\bar{\gamma}^{N \dagger}(K) \leq \frac{T l(K) \bar{S}}{\phi}$ and

$$
\bar{\Gamma}^{\dagger}(K)-\bar{\gamma}^{N \dagger}(K) \geq-\frac{T l(K) \bar{S}}{\phi} .
$$

It hence remains to prove that

$$
K+(1-T) \frac{l(K)}{r(K)} \bar{S}-\frac{T l(K) S}{\phi} \geq 0 \text { for all } K \geq K^{k r} .
$$

To this end, it is sufficient to show

$$
\frac{(1-T)}{r(K)} \geq \frac{T}{\phi}
$$

or equivalently

$$
r(K) \leq \frac{\phi(1-T)}{T} \quad \text { for all } K \geq K^{k r} .
$$

For such $K, r \leq \rho$, so that it remains to verify 


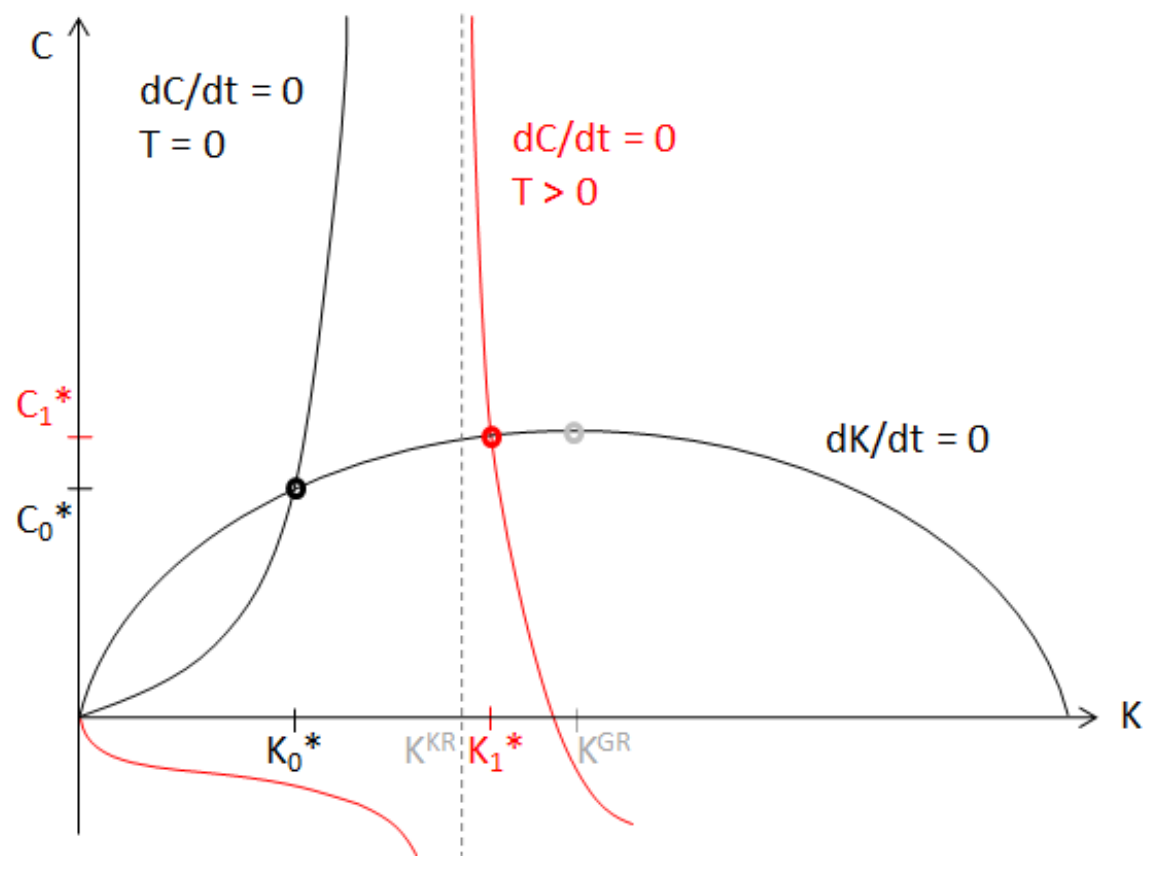

Figure 3: Phase diagram for redistributions with $\bar{\Gamma}^{\dagger}(K)-\bar{\gamma}^{N \dagger}(K)<0$ for some $K \in\left[0, K^{k r}\right]$ and $T>T^{o p t}$

$$
\rho \leq \frac{\phi}{T}-\phi .
$$

The last equation holds if and only if $T \leq \frac{\phi}{\phi+\rho}$, as required.

The two theorems are very general regarding the redistribution scheme, but the generality implies two disadvantages: First, only a comparison between the unregulated market outcome and a policy case is possible. One may also want to know whether a higher tax implies higher welfare in general. Given the uniform redistribution, a higher tax level does in fact imply higher welfare, but this is not necessarily true for all other redistributions. Second, the theorems consider a welfare improvement through a tax only and are not informative about the achievability of the social optimum. In the following, we will derive an optimality condition and give examples for socially optimal redistribution schemes and their corresponding tax rates.

\subsection{Redistribution schemes}

While land rent taxation improves capital accumulation and welfare for a large class of possible redistributions of the tax revenue, we have so far not considered specific redistribution schemes. We state a general optimality 
condition and then discuss four specific redistributions: the transfers are uniform across cohorts of all ages, positive only for newborns, exponential functions of age or wealth-neutral. All particular redistribution schemes discussed are steady-state compatible because of their symmetry.

\subsubsection{General condition for taxes and transfers to newborns for social optimality}

For arbitrary tax levels and redistribution schemes, the capital interest equation 26 generalizes to

$$
r\left(K^{*}\right)=\rho+\phi(\rho+\phi) \frac{K^{*}+p\left(K^{*}, T\right) \bar{S}+\bar{\Gamma}^{*}-\bar{\gamma}^{N *}}{C^{*}} .
$$

Thus, for a combination of a land rent tax and a redistribution scheme to be socially optimal, it is required that $K^{*}+p\left(K^{*}, T\right) \bar{S}+\bar{\Gamma}^{*}-\bar{\gamma}^{N *}=0$ or

$$
K^{*}+\frac{l\left(K^{*}\right) \bar{S}}{r\left(K^{*}\right)}=\frac{T l\left(K^{*}\right) \bar{S}}{r\left(K^{*}\right)}-\bar{\Gamma}^{*}+\bar{\gamma}^{N *} .
$$

Using Equation 27 to replace $\bar{\Gamma}^{*}$, an optimality condition is obtained:

$$
\bar{\gamma}^{N *}=\frac{r\left(K^{*}\right) K^{*}+l\left(K^{*}\right) \bar{S}}{r\left(K^{*}\right)+\phi} .
$$

In terms of aggregate present value the equivalent expression is:

$$
\bar{\Gamma}^{*}=\frac{T l\left(K^{*}\right) \bar{S}}{r\left(K^{*}\right)}-\frac{\phi}{r\left(K^{*}\right)+\phi}\left[K^{*}+\frac{l\left(K^{*}\right) \bar{S}}{r\left(K^{*}\right)}\right] .
$$

To assess the feasibility of a socially optimal fiscal policy, this general condition needs to be evaluated subsequently for specific redistribution schemes $\gamma(\nu, \tau)$. The results are summarized in Table 17

\subsubsection{Uniform distribution}

Under a uniform age-independent transfer scheme, per capita transfers are

$$
\gamma_{u}(t) \equiv T l(t) \bar{S}
$$

\footnotetext{
${ }^{7}$ Although the condition for social optimality defines a steady-state - for any $C, r^{*}=$ $\rho$ is a solution to Equation 23 -, we do not claim stability for this steady-state as it is unknown whether it holds for all redistributions considered below. However, as the redistributions which reach the social optimum also approximate this steady-state arbitarily closely by the (stable) "hyperbolic" steady-state solution of Equation (23), the subsequent sections legitimately define a benchmark for evaluating redistribution schemes.
} 


\begin{tabular}{lcccc}
\hline Distribution & $\bar{\Gamma}^{*}$ & $\bar{\gamma}^{N *}$ & $\begin{array}{c}\text { Condition for } \\
\text { social optimum }\end{array}$ & $\begin{array}{c}\text { Optimal tax level } \\
T^{o p t}\end{array}$ \\
\hline Wealth-neutral & unknown & unknown & impossible & $\mathrm{N} / \mathrm{A}$ \\
\hline Uniform & $\frac{T l^{*} S}{r^{*}+\phi}$ & $\frac{T l^{*} S}{r^{*}+\phi}$ & impossible & $\mathrm{N} / \mathrm{A}$ \\
\hline Newborns only & 0 & $\frac{T l^{*} S}{\phi}$ & $\phi K^{*} \leq l^{*} \bar{S}$ & $\frac{\phi\left(r^{*} K^{*}+l^{*} \bar{S}\right)}{\left(\phi+r^{*}\right) l^{*} \bar{S}}$ \\
\hline Exponential & & & & \\
$\left(\right.$ parameters $\left.a_{0}, a_{s}\right)$ & $\frac{\phi a_{0}}{\left(a_{s}+r^{*}+\phi\right)\left(\phi+a_{s}\right)}$ & $\frac{a_{0}}{a_{s}+r^{*}+\phi}$ & $\phi K^{*}<l^{*} \bar{S}$ & $\frac{\left(r^{*}+\phi\right) a_{0}-r\left(r^{*} K+l^{*} \bar{S}\right)}{a_{0} \phi\left(r^{*} K+l^{*} \bar{S}\right)}$ \\
\hline Capital subsidy & $\mathrm{N} / \mathrm{A}$ & $\mathrm{N} / \mathrm{A}$ & $\phi K^{*} \leq \frac{1}{(\rho+\phi)} \frac{C^{*}}{K^{*}} l^{*} \bar{S}$ & see Equation $\sqrt[53]{53}$ \\
\hline
\end{tabular}

Table 1: Properties of redistributions of land tax revenue in the steady-state: The dependencies of $l^{*}$ and $r^{*}$ on $K$ are suppressed for readability.

Thus, the present value of transfers to individuals and its aggregation over all cohorts have the same value:

$$
\begin{aligned}
& \bar{\gamma}_{u}(t)=\int_{t}^{\infty} T l(\tau) \bar{S} e^{-R(t, \tau)(\tau-t)} \mathrm{d} \tau, \\
& \bar{\Gamma}_{u}(t)=\int_{-\infty}^{t} \bar{\gamma}_{u}(t) \phi e^{\phi(\nu-t)} \mathrm{d} \nu=\bar{\gamma}_{u}(t) .
\end{aligned}
$$

In the steady-state the integrals have an explicit solution:

$$
\bar{\gamma}_{u}(t)^{*}=\bar{\Gamma}_{u}(t)^{*}=\frac{T(t) l^{*} \bar{S}}{r^{*}+\phi} .
$$

One can derive

Proposition 6. Reaching the social optimum is infeasible with the uniform redistribution of tax revenues.

To demonstrate this, insert the values for this specific redistribution into the equations for aggregate consumption and consumption growth:

$$
\frac{\dot{C}(t)}{C(t)}=r(t)-\rho-\phi(\rho+\phi) \frac{K(t)+p(t) \bar{S}}{C(t)} .
$$


In the steady-state, it is also true that

$$
C^{*}=(\rho+\phi)\left[K^{*}+p^{*} \bar{S}+H^{*}+\frac{T l^{*} \bar{S}}{r^{*}+\phi}\right] .
$$

The capital stock dynamics $\dot{K}$ remain unchanged.

While the two distribution-related terms cancel in Equation (35), the effect of the land rent $\operatorname{tax} T>0$ on the land price $p$ remains and leads to a welfare improvement compared to the case without taxation. But even if the price falls to zero for the maximum tax level $T=1$, the last term does not vanish since aggregate growth is still reduced by the newborns' lack of capital.

\subsubsection{Redistribution to newborns only}

Next, the case in which all tax revenues are given to newborn individuals and all others receive nothing is considered:

$$
\gamma_{n}(\nu, t) \equiv \frac{T(t) l(t) \bar{S}}{\phi} \delta(\nu-t) .
$$

Here $\delta(\cdot)$ is a Dirac distribution defined such that

$$
\int_{I} \delta(x) f(x) \mathrm{d} x=\left\{\begin{aligned}
f(0) & \text { if } 0 \in I \\
0 & \text { otherwise }
\end{aligned}\right.
$$

for any continuous function $f: \mathbb{R} \rightarrow \mathbb{R}$ and compact interval $I$.

The present value of transfers to individuals and its aggregation over all cohorts are 8

\footnotetext{
${ }^{8}$ It is instructive to consider two ways of obtaining the latter result, $\bar{\Gamma}=0$. The first is to directly use $\bar{\gamma}_{n}$ in the definition of $\bar{\Gamma}$,

$$
\bar{\Gamma}_{n}(t)=\int_{-\infty}^{t} \int_{t}^{\infty} \frac{T l(\tau) \bar{S}}{\phi} \delta(\nu-t) e^{-R(t, \tau)} \mathrm{d} \tau \phi e^{\phi(\nu-t)} \cdot \mathrm{d} \nu
$$

The inner integral is $T l \bar{S} / \phi$ for $\nu=t$ and zero for $\nu<t$. Unlike the Dirac distribution, the value at $\nu=t$ is finite - thus, the outer integral is zero.

The second approach is to approximate the Dirac distribution by an exponential function (see Section 3.3.4,

$$
\gamma(\nu, t)=G u e^{-u(t-\nu)} \stackrel{u \rightarrow \infty}{\rightarrow} \gamma_{n}(\nu, t) \quad \text { where } \quad G=T l \bar{S} / \phi,
$$

which yields

$$
\bar{\Gamma}_{n}(t)=\frac{G \phi u}{(r+\phi+u)(\phi+u)} \stackrel{u \rightarrow \infty}{\rightarrow} 0 .
$$
}




$$
\begin{aligned}
\bar{\gamma}_{n}(t, t) & =\frac{T(t) l(t) \bar{S}}{\phi}, \\
\bar{\gamma}_{n}(\nu, t) & =0 \quad \text { for } \nu>t \quad \text { and } \\
\bar{\Gamma}_{n}(t) & =0 .
\end{aligned}
$$

In this case, aggregate consumption (11) and consumption growth 13 . become

$$
\begin{aligned}
C(t) & =(\rho+\phi)[K(t)+p(t) \bar{S}+H(t)] \\
\frac{\dot{C}(t)}{C(t)} & =r(t)-\rho-(\rho+\phi) \frac{\phi[K(t)+p(t) \bar{S}]-T(t) l(t) \bar{S}}{C(t)}
\end{aligned}
$$

Again, $\dot{K}$ remains unchanged.

If there exists a tax $T \leq 1$ such that the last term in Equation (43) is zero, the social optimum can be reproduced. That is, the optimal tax in the steady state is

$$
T^{o p t}=\frac{\phi\left(r^{*} K^{*}+l^{*} \bar{S}\right)}{\left(\phi+r^{*}\right) l^{*} \bar{S}} .
$$

Such a tax and thus the social optimum is feasible, if there exists a tax level $T \leq 1$. Thus we have proved

Theorem 7 (Feasibility of the social optimum). The socially optimal outcome can be implemented with a land rent tax and a redistribution of the tax revenue to only the newborns if

$$
\phi K^{k r} \leq l^{k r} \bar{S} .
$$

This is an intuitive result, stating that a tax and targeted redistribution achieves the social optimum if the (originally) missing capital of the newborns is smaller than the transfers that they may receive - which is, at the maximum, the entire land rent. So the negative aggregate consumption effect of the former can be compensated by the latter.

The result also gives an absolute bound for reaching the social optimum in our model: in continuous OLG models, underaccumulation is the result of a lack of wealth of the newborns; thus redistributing to that generation the full revenue is the most efficient way of curing the inefficiency (if the revenue is so high that it leads to overaccumulation, the tax rate can be lowered). This will be more transparent by considering a redistribution based on a function that approximates the Dirac function chosen in this section. 


\subsubsection{Exponential redistribution schemes}

For assessing the robustness of the condition on the feasibilitiy of the social optimum, it is instructive to consider redistributing the land tax revenue by an exponential function in age approximating the Dirac distribution 9 This redistribution has two parameters: $a_{0}$ denotes the value of the redistribution at birth and $a_{s}$ denotes the speed of the exponential change with age. The exponential redistribution scheme depending on $a_{0}$ and $a_{s}$ is then defined by

$$
\gamma_{e}(\nu, \tau)=a_{0} e^{-a_{s}(\tau-\nu)} .
$$

For this redistribution to be permissible in the sense of Definition 1, a restriction on the choice of $a_{0}$ and $a_{s}$ is required:

$$
T l^{*} \bar{S}=\frac{a_{0} \phi}{\left(a_{s}+\phi\right)} \quad \text { with }\left(a_{s}+\phi\right)>0 .
$$

The restriction is obtained by solving the integral in Equation (4) for $\gamma_{e}$. It implies that $a_{0}$ is positive and that $a_{s}>-\phi$. So for $-\phi<a_{s}<0$ an exponential increase with age is permissible, but it can be shown that it cannot be socially optimal. For finding a condition for social optimality it can instead be calculated that

$$
\begin{aligned}
\bar{\gamma}_{e}^{*} & =\frac{a_{0}}{r^{*}+\phi+a_{s}} \quad \text { and } \\
\Gamma_{e}^{*} & =\frac{\phi a_{0}}{\left(r^{*}+\phi+a_{s}\right)\left(\phi+a_{s}\right)} .
\end{aligned}
$$

To determine when the social optimum can be reached by this redistribution, Equations (32), 47) and (48) need to be combined to calculate $a_{0}$ and $a_{s}$ explicitly. It can be shown that

$$
a_{0}=\frac{T l^{*} \bar{S} r^{*}\left(r^{*} K^{*}+l^{*} \bar{S}\right)}{T l^{*} \bar{S}\left(r^{*}+\phi\right)-\phi\left(r^{*} K^{*}+l^{*} \bar{S}\right)}
$$

whence $a_{0}$ is positive if the denominator is. Setting $T=1$, it is proved that

Proposition 8. A redistribution scheme in which land rents are given back to the households according to an exponential function decreasing in age can reach the social optimum if

$$
l(t) \bar{S}>\phi K .
$$

The result confirms that the exponential function used approximates the Dirac distribution. Moreover, if social optimality is feasible, the optimal tax is then

$$
T^{o p t}=\frac{\left(r^{*}+\phi\right) a_{0}-r^{*}\left(r^{*} K+l^{*} \bar{S}\right)}{a_{0} \phi\left(r^{*} K+l^{*} \bar{S}\right)} .
$$

\footnotetext{
${ }^{9}$ We are indebted to Dankrad Feist for suggesting this redistribution.
} 


\subsubsection{Wealth-neutral redistribution}

The analysis of tax incidence requires compensation of individuals to isolate the effect of the tax as a shift in relative prices Calvo, Kotlikoff and Rodriguez 1979. For the model in this study, it can be shown that land rent taxation combined with wealth-neutral redistribution of revenues to individuals cannot establish the social optimum and even yields lower welfare than a uniform redistribution scheme. In the following, a sketch of the argument is presented.

Starting from the uniform redistribution, some of the transfers from some selected young generations are shifted to selected older generations, which will have accumulated more land and thus have a higher land rent tax burden. At time $\tilde{t}$, the shift of contemporaneous transfers does not have an effect on aggregate consumption (Equation (11)) since any cohort consumes the same fraction of their wealth. However, the expectation of future transfers of the same pattern does have an effect, since the expected increased transfers towards today's youngest generations will be at the cost of unborn generations $(\nu>\tilde{t})$, whose future loss finances today's consumption. Technically, $\bar{\Gamma}(\tilde{t})$ is higher than without the shift. By itself, this effect increases aggregate consumption at any given capital stock level - the hyperbola described by Equation (23) with shifted transfers is above the original hyperbola for all values of the capital stock. ${ }^{10}$ However, since higher aggregate consumption implies foregone investment and thus a lower steady state capital stock, the overall effect on the steady-state level of aggregate consumption is negative. Thus, since the uniform redistribution, from which we started, is not socially optimal, the wealth-neutral redistribution which gives more transfers to older cohorts owning more land can neither be optimal.

\subsection{Capital subsidy}

An alternative to redistributing tax revenues directly to individuals is to subsidize capital in the form of a markup on the market interest rate. This does not change the results in Section 2 except that $r$ is replaced by $\tilde{r} \equiv r+\epsilon$, with $\epsilon$ being the markup financed by land rent tax revenues. Specifically, aggregate consumption growth becomes

$$
\dot{C}(t)=C(t)[r(t)+\epsilon-\rho]-\phi(\rho+\phi)[K(t)+p(t) \bar{S}],
$$

so restoring the Keynes-Ramsey case requires

$$
\epsilon C=\phi(\rho+\phi)(K(t)+p(t) \bar{S}) .
$$

\footnotetext{
${ }^{10}$ Additionally, $\bar{\gamma}^{N}$ is lower when transfers are shifted, since increased transfers in the far future are discounted more than losses in the nearer future. This strengthens the overall effect of the shift of transfers, $\left(\bar{\Gamma}^{*}-\bar{\gamma}^{N *}\right)$ in Equation 23 .
} 
Using $\epsilon K^{*}=T l\left(K^{*}\right) \bar{S}$ and $p^{*}=(1-T) l\left(K^{*}\right) / r^{*}$, a steady state condition for the optimal tax is obtained:

$$
\left[\frac{l^{*} \bar{S}}{K^{*}}+\phi(\rho+\phi) \frac{l^{*} \bar{S}}{r^{*} C^{*}}\right] T^{o p t}=\phi(\rho+\phi)\left[\frac{K^{*}}{C^{*}}+\frac{l^{*} \bar{S}}{r^{*} C^{*}}\right] .
$$

Hence a proposition on the feasibility of the social optimum for the capital subsidy can be deduced by inserting $T \leq 1$ :

Proposition 9. Reaching the social optimum with a tax on land rents to finance a capital subsidy is feasible if

$$
\phi K^{*} \leq \frac{1}{(\rho+\phi)} \frac{C^{*}}{K^{*}} l^{*} \bar{S} .
$$

\section{Discussion}

We subsequently discuss the empirical relevance of our theoretical result for fiscal policy and also delineate its normative validity. To this end we first present a rough estimate indicating that achieving the social optimum by a capital subsidy or transfers to newborns is empricially feasible (Section 4.1). Second, we detail under which conditions the suggested policy instruments are desirable (Section 4.2). We finally outline potential modifications and extensions of our framework, notably possibilities of financing public capital with the land rent tax revenue (Section 4.3).

\subsection{Empirical Relevance}

Can our conditions for implementing the social optimum be fulfilled in practice? First we consider the feasibility of reaching the social optimum by redistributing all land tax revenues to the newborns, as in Section 3.3.3. For the rough estimate, assume a Cobb-Douglas production function

$$
Y=F(L, K, S)=F_{0} L^{(1-\alpha-\beta)} K^{\alpha} S^{\beta},
$$

so that $l^{*} \bar{S}=\beta Y^{*}$. Denoting the steady-state ratio of the total capital stock to total output by $\kappa=\frac{K^{*}}{Y^{*}}$, the feasibility condition 45 becomes

$$
\phi \kappa \leq \beta .
$$

We use data from Caselli and Feyrer 2007 for $\kappa$ and to approximate $\beta$. ${ }^{11}$ The dataset covers a wide variety of countries, ranging from Côte d'Ivoire and Peru to Switzerland and the USA. We find that the feasibility condition

\footnotetext{
${ }^{11}$ Caselli and Feyrer Caselli and Feyrer 2007 do not report $\beta$ directly, but estimates of "one minus the labor share" (p.541) in income and the share of reproducible capital in income. The difference - our approximation for $\beta$ - is the income share of land and other natural resources, some of which are not fixed factors. However, the authors report
} 
is satisfied with realistic values of $\phi$ for all 53 countries quoted, often by a wide margin ${ }^{12}$ For the Cobb-Douglas case, the optimal steady state tax is

$$
T_{C D}^{o p t}=\frac{\phi \kappa \alpha+\phi \kappa \beta}{\alpha \beta+\phi \kappa \beta} .
$$

This implies that the lower $\kappa$ and the higher $\beta$, the lower the share of the land rent that has to be redistributed to the newborns.

Second, for the feasibility of a social optimum by capital subsidies from Section 3.4, again assume the Cobb-Douglas production function given by Equation (55) and note that from the total capital equation (12), we have $\dot{K}=Y-\delta K-C=0$ in the steady state. Then, the feasibility condition (54) becomes

$$
\phi \kappa \leq \frac{1}{(\rho+\phi)}\left(\frac{1}{\kappa}-\delta\right) \beta
$$

Even if we assume high values for the additional parameters in this equation - for instance $\rho=0.05$ and $\delta=0.15-$, we find that this feasibility condition is weaker than for the case of transfers by a factor of two or more for the 53 countries quoted Caselli and Feyrer 2007. It is weaker by a factor of 10 and more if we assume $\rho=0.01$ and a more realistic depreciation rate $\delta=0.05$.

\subsection{Normative cogency}

In this study we evaluated policy options by reference to two normative viewpoints that are equivalent in the present context. Either the social planner is a preference-satisfaction utilitarian in the sense of [Calvo and Obstfeld 1988] who for the time being only cares about the dynamically optimal allocation or she wishes to create a society with perfect altruism between generations.

\footnotetext{
"Proportions of different types of wealth in total wealth" (p.547) which demonstrate that while subsoil resources are important for some countries (their mean wealth share is $10.5 \%$, with a standard deviation of 16.4, compared to a 34.8\% mean share of land-related wealth), land wealth dominates in most cases (subsoil resources' median wealth share is only $1.5 \%$, compared to a $23.5 \%$ median share of land-related wealth). Since the dataset does not include any countries that mainly rely on fossil fuel extraction, such as countries on the Arabic Peninsula, and given the wide margin by which the sufficiency condition is fulfilled for most countries (see below), we consider this rough approximation as sufficient for our purposes.

${ }^{12}$ For example, Switzerland has the highest $\kappa=3.59$ and lowest $\beta=0.06$ in the dataset Caselli and Feyrer 2007, so we need $\phi \leq 0.017$. The real birth rate is 0.010 Eurostat 2012, so there is even scope to accomodate modest population growth (the death rate is 0.008 Eurostat 2012. Also, $\phi$ is lower than the real birth rate because in reality there are some bequests. For comparison, the USA have $\kappa=2.19$ and $\beta=0.08$ Caselli and Feyrer 2007, implying $\phi \leq 0.037$. Most other developed countries in the dataset range between Switzerland and the USA, while most industrialising and developing countries have lower capital-to-output ratios and higher shares of land in output (e.g. Morocco with $\kappa=1.31, \beta=0.19$ and thus $\phi \leq 0.145$, which would allow sufficient transfers to newborns even for a high population growth rate).
} 
Throughout we compare an existing steady-state without policy and a new steady-state with policies in place that results from the existing one. So some agents will live in the conditions of both states ${ }^{13}$ Here we point to three limitations of these justifications of the fiscal policies we suggest.

First, our analysis only compares the welfare outcome in steady-states while we did not consider transitional dynamics. This means that our policies are supposedly no Pareto-improvements as some generations might be worse off during the transition from a steady-state without policies to one with a policy in place. Smoothing the transition for all generations to find a Pareto-improving tax policy is likely to involve an elaborate scheme of lump-sum transfers Heijdra and Meijdam 2002.

Second, even if aggregate welfare is higher in one steady-state than another, our suggested redistribution schemes are not Pareto-improving when comparing steady-states only: older generations - "rentiers" - will be worse off in some cases. However, the fact that aggregate welfare is higher in all suggested redistribution schemes implies that our policies satisfy the Kaldor-Hicks criterion Hicks 1940, Kaldor 1939], that is, constitute a potential Pareto-improvement.

Third, we emphasize that a preference-satisfaction social optimum would only be achieved if also the static, not only the dynamic optimum were implemented. Picking a redistribution of rents that also implements the statically optimal distribution seems possible, but whether this can be achieved without practically infeasible lump-sum taxes is an open problem for further work.

We note that for one redistribution that we have examined, the last two caveats do not exist. The wealth-neutral redistribution (see Section 3.3.5 is a Pareto-improvement in the steady-state (although it is not socially optimal).

What does this mean in practice? While we identify in this paper empirically plausible possibilities for increasing total welfare by a land rent tax, the redistribution scheme chosen by a government would also need to take into account transitional dynamics and political feasibility given that rentiers may be made worse-off.

\subsection{Extensions and Modifications}

Three ways to further examine the validity of hypergeorgism in different contexts naturally suggest themselves.

First, it would be desirable to study the implications of the fiscal policies suggested above not only by comparing steady states, but also by taking account of the transitional dynamics between a steady state without the policies and one with the policies in place. Such an analysis would need

\footnotetext{
${ }^{13}$ If instead one compared two separate dynamical systems, all welfare-improving policies would also be Pareto improving, but that case is not politically relevant.
} 
to follow the approach taken by Heijdra and Meijdam 2002 for the case of financing productive public capital.

Second, it should be analyzed whether hypergeorgism is also a valid theory for other causes of underaccumulation of capital than that implicit in the continuous OLG-model. While in this study the reason for underaccumulation was assumed to be imperfect altruism between generations resulting in the perpetual appearance of fundless 'newborns', further potential causes of underaccumulation are vital to consider for a sound assessment of fiscal policy: on the side of the firms, capital may be allocated inefficiently in the investment process [Scharfstein and Stein 2000, the social value of uninternalized spillovers might not be realized [Romer 1986] or capital markets themselves may be inefficient [Fama 1970, Fama 1991]. But even on the side of the households, a further possible cause of underaccumulation is that in general real human decision-makers are known to have time-inconsistent preferences as they discount the future hyperbolically Loewenstein and Prelec 1992, Laibson 1997, Frederick, Loewenstein and O'Donoghue 2002. In particular they have self-control problems concerning savings which at least in the context of the U.S. economy is assumed to lead to underinvestment [Thaler and Benartzi 2004, Benartzi and Thaler 2007.

Third, the cause for land rent taxation could be strengthend by examining the possibility of using its non-distortionary revenue for reducing other inefficiencies than suboptimal capital accumulation, notably investments in productive public capital. For the case of a single dynastic household, our companion paper Mattauch, Siegmeier and Edenhofer 2013 provides some results that can be extended to the setting of overlapping generations (see also Heijdra and Meijdam 2002 ). While using the tax revenue for investment in public capital will constitute a welfare-improvement, this will not generally be socially optimal if no further revenue is left for redistributive transfers to the newborns. Only if the land rent exceeds the sum of the socially optimal investment in public capital and the miminum amount $\phi K$ required for curing the inefficient capital accumulation can the social optimum be reproduced. The content of the neoclassical Henry George Theorems is that in some circumstances confiscating (land) rents is sufficient for financing the optimal level of a public good Stiglitz 1977]. The suggested analysis would be an extension of that content to the context of intertemporal infrastructure financing with a redistributive twist.

Taking these extensions together, a more complete picture should emerge of how generating revenue from land rent taxation can enhance economic prosperity. We hope to adress some of these extensions in further work. 


\section{Conclusion}

This paper studied the welfare effect of land rent taxation and how the revenues should be redistributed to a population of heterogeneous households with imperfect intergenerational altruism. It was shown that taxing land rents leads to an increase in aggregate consumption and thus a potential increase in welfare, as long as the revenue is not recycled in a too redistributive way when the land rent tax is high. While the government cannot implement the social optimum with a wealth-neutral or a uniform redistribution, giving high transfers to the youngest generations or subsidising productive capital are potentially socially optimal policies. Achieving the social optimum by such policies is possible as long as the total land rent is greater than the stock of productive capital multiplied by the birth rate, a condition which could be confirmed for a diverse set of countries. In summary, our findings support the view that under imperfections in the accumulation of productive assets, taxing and redistributing rents on fixed production factors is a policy measure that leads to a welfare gain - a view we label hypergeorgism.

\section{Appendix}

\subsection{Derivation of the Keynes-Ramsey rule and the arbitrage condition}

The budget constraint (5) can be split into a constraint on monetary terms and a constraint on land size by defining $d(\nu, t)=\phi s(\nu, t)-\dot{s}(\nu, t)$. Dropping the time arguments, we obtain:

$$
\begin{aligned}
\dot{k} & =w+[r+\phi] k+(1-T) l s+p d+\gamma-c \\
\dot{s} & =\phi s-d .
\end{aligned}
$$

Individuals maximize utility given by Equation (1) by choosing $c(\nu, \tau)$ and $d(\nu, \tau)$, subject to Equations (56), (57) and the transversality condition (6). Writing $\lambda$ and $\mu$ for the multipliers of (56) and (57) in the current value Hamiltonian $H_{c}$, we obtain the following first order conditions:

$$
\begin{aligned}
\frac{\partial H_{c}}{\partial c} & =\frac{1}{c}-\lambda=0 \\
\frac{\partial H_{c}}{\partial d} & =\lambda p-\mu=0 \\
\frac{\partial H_{c}}{\partial k} & =(\rho+\phi) \lambda-\dot{\lambda} \\
\Rightarrow \lambda(r+\phi) & =(\rho+\phi) \lambda-\dot{\lambda} \\
\frac{\partial H_{c}}{\partial s} & =\lambda(1-T) l+\mu \phi \\
\Rightarrow \lambda(1-T) l+\mu \phi & =(\rho+\phi) \mu-\dot{\mu} .
\end{aligned}
$$


Inserting the time derivative of $(58)$ into Equation 60 yields the KeynesRamsey rule (7). Using Equation (59) and its time derivative to replace $\mu$ and $\dot{\mu}$ in Equation (61) and applying Equation (60) gives the arbitrage condition for investing in land or capital (8).

\subsection{Individual lifetime budget constraint and consumption level}

First, the lifetime budget constraint $(9)$ is derived, from which the individual consumption level can then be obtained. Dropping the time arguments $\nu$ and $\tau$ where no confusion is possible, regrouping terms in (5) and adding $\dot{p} s-(r+\phi) p s$ on both sides, it follows that:

$$
\begin{aligned}
\dot{k}+p \dot{s}+\dot{p} s-(r+\phi)(k+p s) & =w+(1-T) l s+\gamma+\dot{p} s-r p s-c= \\
& =w+\gamma-c
\end{aligned}
$$

The last equality follows from (8). This leads to

$$
\begin{aligned}
\frac{d}{d \tau}\left[(k+p s) e^{-R}\right] & =(w+\gamma-c) e^{-R} \\
\Rightarrow \int_{t}^{\infty} \frac{d}{d \tau}\left[(k+p s) e^{-R}\right] \mathrm{d} \tau & =\int_{t}^{\infty}(w+\gamma-c) e^{-R} \mathrm{~d} \tau .
\end{aligned}
$$

For the integral on the left-hand side, note that $\exp (-R(t, t))=1$ and use (6) to obtain

$$
\begin{aligned}
& \int_{t}^{\infty} \frac{d}{d \tau}\left[(k+p s) e^{-R}\right] \mathrm{d} \tau= \\
= & \lim _{\tau \rightarrow \infty}\left([k(\nu, \tau)+p(\tau) s(\nu, \tau)] e^{-R(t, \tau)}\right)-k(\nu, t)-p(t) s(\nu, t)= \\
= & -k(\nu, t)-p(t) s(\nu, t) .
\end{aligned}
$$

Using the definition of $H(t)$ and $\bar{\gamma}(\nu, t)$ from the main text, the right-hand side can be written as

$$
\int_{t}^{\infty}(w+\gamma-c) e^{-R} \mathrm{~d} \tau=H(t)+\bar{\gamma}(\nu, t)-\int_{t}^{\infty} c(\nu, \tau) e^{-R} \mathrm{~d} \tau .
$$

Combining equations $(62)$ and $(63)$, we obtain the lifetime budget constraint (9).

Then, the individual consumption level follows in two steps. First, solve the Keynes-Ramsey rule for $c$,

$$
\begin{aligned}
77) & \Rightarrow \int_{c\left(\nu, t_{0}\right)}^{c(\nu, \bar{t})} \frac{1}{c(\nu, \tau)} \mathrm{d} c=\int_{t_{0}}^{\bar{t}}(r(\tau)-\rho) \mathrm{d} \tau \\
& \Rightarrow c(\nu, \bar{t})=c\left(\nu, t_{0}\right) \exp \left(\int_{t_{0}}^{\bar{t}}(r(\tau)-\rho) \mathrm{d} \tau\right) .
\end{aligned}
$$


Second, setting $t_{0}=t$ and $\bar{t}=\tau$ in the last expression and replacing $c$ in the lifetime budget equation,

$$
\begin{aligned}
k(\nu, t)+p(t) s(\nu, t)+H(t)+\bar{\gamma}(\nu, t) & =\int_{t}^{\infty} c(\nu, t) e^{\int_{t}^{\tau}[r(\tilde{t})-\rho] \mathrm{d} \tilde{t}} e^{-R(t, \tau)} \mathrm{d} \tau= \\
& =c(\nu, t) \int_{t}^{\infty} e^{-\int_{t}^{\tau}(\rho+\phi) \mathrm{d} \tilde{t}} \mathrm{~d} \tau= \\
& =c(\nu, t) /(\rho+\phi) .
\end{aligned}
$$

Thus, the individuals' level of consumption is a fixed fraction of wealth independent of time or the individual's age.

\subsection{Aggregate solution}

We derive the aggregate quantity for general age-dependent transfers $\gamma(\nu, t)$ as given in Section 2 .

The aggregate consumption level $C(t)$ for general transfers is obtained directly from aggregation of Equation (10), as given by Equation (11) in the main text.

The dynamics of the total capital stock 12 are obtained by applying Leibniz' rule to

$$
K(t)=\int_{-\infty}^{t} k(\nu, t) \phi e^{\phi(\nu-t)} \mathrm{d} \nu,
$$

replacing $\dot{k}$ by its expression from the individual budget constraint $(5)$, and using Equation (3) for aggregate changes in land ownership:

$$
\begin{aligned}
\dot{K}(t) & =\underbrace{k(t, t)}_{=0} \phi e^{\phi(t-t)}-0+\int_{-\infty}^{t} \frac{\mathrm{d}}{\mathrm{d} t}\left[k(\nu, t) \phi e^{\phi(\nu-t)}\right] \mathrm{d} \nu= \\
& =-\phi K(t)+\int_{-\infty}^{t} \dot{k}(\nu, t) \phi e^{\phi(\nu-t)} \mathrm{d} \nu= \\
& =w(t)+r(t) K(t)+[1-T(t)] l(t) \bar{S}+ \\
& +p(t) \underbrace{\left[\phi \bar{S}-\int_{-\infty}^{t} \dot{s}(\nu, t) \phi e^{\phi(\nu-t)} \mathrm{d} \nu\right]}_{=0}-C(t)+\underbrace{\int_{-\infty}^{t} \gamma(\nu, t) \phi e^{\phi(\nu-t)} \mathrm{d} \nu}_{=T(t) l(t) \bar{S}}= \\
& =w(t)+r(t) K(t)+l(t) \bar{S}-C(t) .
\end{aligned}
$$

The government budget constraint (4) was used in the last step, so taxes and transfers always cancel out in the last step and the result does not directly depend on the redistribution $\gamma(\nu, t)$. However, it may have an indirect effect via prices, stock levels and consumption. 
Similarly, we derive the dynamics of aggregate consumption given by Equation (13):

$$
\begin{aligned}
\dot{C}(t) & =c(t, t) \phi e^{\phi(t-t)}-0+\int_{-\infty}^{t} \frac{\mathrm{d}}{\mathrm{d} t}\left[c(\nu, t) \phi e^{\phi(\nu-t)}\right] \mathrm{d} \nu= \\
& =\phi(\rho+\phi)[H(t)+\bar{\gamma}(t, t)]-\phi C(t)+\underbrace{\int_{-\infty}^{t} \dot{c}(\nu, t) \phi e^{\phi(\nu-t)} \mathrm{d} \nu}_{=(r(t)-\rho) C(t)}= \\
& =[r(t)-\rho] C(t)-\phi(\rho+\phi)[K(t)+p(t) \bar{S}+\bar{\Gamma}(t)-\bar{\gamma}(t, t)] .
\end{aligned}
$$

The first equality follows from Leibniz' rule. For the second, $c(t, t)=(\rho+$ $\phi)[k(t, t)+p(t) s(t, t)+H(t)+\bar{\gamma}(t, t)]=(\rho+\phi)[H(t)+\bar{\gamma}(t, t)]$ is used. In the third step, $\phi C(t)$ is replaced using Equation (11).

\subsection{Proof of Lemma 2}

Proof. The steady state condition $(27)$ is calculated from $d \bar{\Gamma}^{*} / d t=0$. Applying Leibniz' rule yields:

$$
\begin{aligned}
0=\frac{d \bar{\Gamma}(t)}{d t} & =\frac{d}{d t} \int_{-\infty}^{t} \bar{\gamma}(\nu, t) \phi e^{-\phi(t-\nu)} \mathrm{d} \nu= \\
& =\phi \bar{\gamma}(t, t)-0+\int_{-\infty}^{t} \frac{\partial}{\partial t}\left[\bar{\gamma}(\nu, t) \phi e^{-\phi(t-\nu)}\right] \mathrm{d} \nu= \\
& =\phi \bar{\gamma}^{N}(t)-\phi \bar{\Gamma}(t)+\int_{-\infty}^{t} \frac{\partial \bar{\gamma}(\nu, t)}{\partial t} \phi e^{-\phi(t-\nu)} \mathrm{d} \nu
\end{aligned}
$$

The differentiation under the integral in the last term can be treated similarly for the steady-state interest rate $r^{*}$ :

$$
\begin{aligned}
\frac{\partial \bar{\gamma}(\nu, t)}{\partial t} & =\frac{\partial}{\partial t} \int_{t}^{\infty} \gamma(\nu, \tau) e^{-\left(r^{*}+\phi\right)(\tau-t)} \mathrm{d} \tau= \\
& =0-\gamma(\nu, t)+\int_{t}^{\infty} \frac{\partial}{\partial t}\left[\gamma(\nu, \tau) e^{-\left(r^{*}+\phi\right)(\tau-t)}\right] \mathrm{d} \tau= \\
& =\left(r^{*}+\phi\right) \bar{\gamma}(\nu, t)-\gamma(\nu, t) .
\end{aligned}
$$

Inserting this into Expression (64) above, it follows that

$$
\begin{aligned}
0=\frac{d \bar{\Gamma}(t)}{d t} & \left.=\phi \bar{\gamma}^{N}(t)-\phi \bar{\Gamma}(t)+\left(r^{*}+\phi\right) \bar{\Gamma}(t)-\int_{-\infty}^{t} \gamma(\nu, t) \phi e^{-\phi(t-\nu}\right) \mathrm{d} \nu \\
& =\phi \bar{\gamma}^{N}(t)+r^{*} \bar{\Gamma}(t)-T l \bar{S}
\end{aligned}
$$




\section{Acknowledgements}

We are indebted to Dankrad Feist for sharing his insights on solving systems of integral equations with us. We also wish to thank Olga Heismann, Ole Schwen and Alexandra Surdina for helpful comments concerning the mathematical details of the paper. Financial support from the MichaelOtto-Stiftung for the chair Economics of Climate Change at TU Berlin is gratefully acknowledged. Linus Mattauch thanks the German National Academic Foundation for financial support through a doctoral scholarship.

\section{References}

[Benartzi and Thaler 2007] Benartzi, Shlomo and Richard Thaler (2007). Heuristics and Biases in Retirement Savings Behavior. Journal of Economic Perspectives 21(3): 81-104.

[Blanchard 1985] Blanchard, Olivier (1985). Debts, deficits, and finite horizons. Journal of Political Economy 93: 223-247.

[Buiter 1988] Buiter, Willem H. (1988). Death, Birth, Productivity Growth and Debt Neutrality. The Economic Journal 98: 279-293.

[Buiter 1989] Buiter, Willem H. (1989). Debt neutrality, Professor Vickrey and Henry George's 'single tax'. Economics Letters 29: 43-47.

[Burgstaller 1994] Burgstaller, André (1994). Property and prices: Toward a unified theory of value. Cambridge: Cambridge University Press.

[Calvo, Kotlikoff and Rodriguez 1979] Calvo, Guillermo A., Laurence J. Kotlikoff and Carlos Alfredo Rodriguez (1979). The Incidence of a Tax on Pure Rent: A New (?) Reason for an Old Answer. Journal of Political Economy 87(4): 869-874.

[Calvo and Obstfeld 1988] Calvo, Guillermo A. and Maurice Obstfeld (1988). Optimal time-consistent policy with finite lifetimes. Econometrica 56(2): 411-432.

[Caselli and Feyrer 2007] Caselli, Franceso and James Feyrer (2007). The marginal product of capital. The Quarterly Journal of Economics 122(2): 535-568.

[Chamley and Wright 1987] Chamley, Christophe and Wright, Brian D. (1987). Fiscal incidence in an overlapping generations model with a fixed asset. Journal of Public Economics 32: 3-24.

[Eurostat 2012] Eurostat (2012). Population data retrieved from http://epp.eurostat.ec.europa.eu/ on November 14th 2012. 
[Fama 1970] Fama, Eugene F. (1970). Efficient capital markets: A review of theory and empirical work. The Journal of Finance 25(2): 383-417.

[Fama 1991] Fama, Eugene F. (1991). Efficient capital markets: II. The Journal of Finance 46(5): 1575-1617.

[Feldstein 1977] Feldstein, Martin S. (1977). The surprising incidence of a tax on pure rent: A new answer to an old question. Journal of Political Economy 85(2): 349-360.

[Fried and Howitt 1988] Fried, Joel and Peter Howitt (1988). Fiscal deficits, international trade and welfare. Journal of International Economics 24: $1-22$.

[Foley and Michl 1999] Foley, Duncan K. and Thomas R. Michl (1999). Growth and distribution. Harvard University Press, Cambridge and London.

[Frederick, Loewenstein and O'Donoghue 2002] Frederick, Shane, George Loewenstein and Ted O'Donoghue (2002). Time Discounting and Time Preference. A Critical Review. Journal of Economic Literature 40: 351-401.

[George 1879] George, Henry (1879/1894/2002). Progress and Poverty: An Inquiry into the Cause of Industrial Depressions and of Increase of Want with Increase of Wealth: The Remedy . Chestnut Hill: Adamant Media Corporation.

[Hashimoto and Sakuragawa 1998] Hashimoto, Hiroyuki and Masaya Sakuragawa (1998). Land-tax, transfer and growth in an endogenously growing economy with overlapping generations. Japanese Economic Review 49(4): 412-425.

[Heavey 2003] Heavey, Jerome F. (2003). Comments on Warren Samuels's "Why the Georgist Movement Has Not Succeeded" The American Journal of Economics and Sociology 62(3): 593-599.

[Heijdra 2009] Heijdra, Ben J. (2009). Foundations of modern macroeconomics. Second edition. Oxford University Press, Oxford and New York.

[Heijdra and Meijdam 2002] Heijdra, Ben J. and Lex Meijdam (2002). Public investment and intergenerational distribution. Journal of Economic Dynamics and Control 26: 707-735.

[Stiglitz 1977] Stiglitz, Joseph (1977). The theory of local public goods. In: Martin S. Feldstein and Robert P. Inman (eds). The Economics of Public Services. London: MacMillan. 
[Hicks 1940] Hicks, John R. (1940). The valuation of the social income. Economica 7: 105124.

[Kaldor 1939] Kaldor, Nicolas (1939). Welfare propositions of economics and interpersonal comparisons of utility. The Economic Journal 49: 549-552.

[Laibson 1997] Laibson, David (1997). Golden Eggs and Hyperbolic Discounting. The Quarterly Journal of Economics 112(2): 443-478.

[Loewenstein and Prelec 1992] Loewenstein, George and Drazen Prelec (1992). Anomalies and Intertemporal Choice: Evidence and an Interpretation. Quarterly Journal of Economics 107(2): 573-597.

[Mattauch, Siegmeier and Edenhofer 2013] Mattauch, Linus, Jan Siegmeier and Ottmar Edenhofer (2013). Financing public capital through rent taxation. mimeo.

[Petrucci 2006] Petrucci, Alberto (2006). The incidence of a tax on pure rent in a small open economy (2006). Journal of Public Economics 90(4-5): 921-933.

[Romer 1986] Romer, Paul A. (1986). Increasing Returns and Long-Run Growth. The Journal of Political Economy 94(5): 1002-1037.

[Scharfstein and Stein 2000] Scharfstein, David S. and Jeremy C. Stein (2000). The Dark Side of Internal Capital Markets: Divisional RentSeeking and Inefficient Investment. The Journal of Finance 55(6): 2537-2564.

[Thaler and Benartzi 2004] Thaler, Richard and Shlomo Benartzi (2004). Save more tomorrow ${ }^{\mathrm{TM}}$ : Using Behavioral Economics to Increase Employe Saving. Journal of Political Economy 112(S1): S164-S185.

[Weil 1989] Weil, Philippe (1989). Overlapping families of infinitely-lived agents. Journal of Public Economics 38: 183-198.

[Yaari 1965] Yaari, Menahem E. (1965). Uncertain lifetime, life insurance and the theory of the consumer. Review of Economic Studies 32: 137150 . 